\title{
Mutual coherence of optical and matter waves
}

\author{
G.A. Prataviera, E. V. Goldstein, and P. Meystre \\ Optical Sciences Center, University of Arizona, Tucson, AZ 85721
}

\begin{abstract}
We propose a scheme to measure the cross-correlations and mutual coherence of optical and matter fields. It relies on the combination of a matter-wave detector operating by photoionization of the atoms and a traditional absorption photodetector. We show that the double-detection signal is sensitive to cross-correlation functions of light and matter waves.
\end{abstract}

PACS numbers: 03.65.Bz, 03.75.-b, 42.50.-p

\section{INTRODUCTION}

The manipulation and control of matter waves by optical fields forms a new paradigm that is now receiving much attention. In a first generation of experiments, externally applied optical fields were used to manipulate ultracold atomic samples. Examples include optical dipole traps for multicomponent condensates [1] and the diffraction of condensates by optical gratings [2,3]. More recent experiments are also concerned with the back-action of the condensate on the light field. For example, Bragg scattering of light off a condensate has been used to determine its form factor 佂, and recently, superradiance experiments [5] have demonstrated the joint build-up of optical and matter-wave fields. One topic of particular interest in this context is the possibility to generate crosscorrelations and quantum entanglement between optical and matter waves. Indeed, there are already several theoretical predictions of such effects [6 9], e.g. in an ultracold version of the Collective Atom Recoil Laser [10,11.

It is therefore timely to investigate ways to measure the joint quantum statistical properties of optical and matter-wave fields. This is an extension from the usual quantum optics situation, where one normally measures either the statistical properties of light, or more rarely, those of the atoms, but not joined properties of the coupled system. For this latter purpose, it is necessary to develop a generalized detection theory applicable to the simultaneous detection of optical and matter-wave fields.

Our approach builds on the well-known results of optical detection and coherence theory [12 and their recent extension to atomic matter waves [13]. Specifically, we consider a detection scheme consisting of an atomic detector operating by ionization 13] and a traditional photodetector operating by absorption 12, 14. Our model is introduced in section II. Section III presents general expressions for the correlations between optical and matter fields. Our general results are illustrated in section IV, where mutual coherence functions are calculated for a simple model of linear coupling between optical and matter waves. Finally, section V is a summary and conclusion.

\section{THE DETECTION SCHEME}

We consider a situation where an optical field and an atomic matter-wave field are dynamically coupled, and we want to quantify the cross-correlation and mutual coherence of these fields. The matter-wave field might, but needs not to be, a Bose-Einstein condensate, described by a multicomponent field operator $\boldsymbol{\Psi}(\mathbf{r})$, satisfying the bosonic commutation relations

$$
\left[\Psi_{i}(\mathbf{r}), \Psi_{j}^{\dagger}\left(\mathbf{r}^{\prime}\right)\right]=\delta_{i j} \delta\left(\mathbf{r}-\mathbf{r}^{\prime}\right)
$$

where $\mathbf{r}$ is the center-of-mass coordinate of the atoms and the indices $i$ label their internal state. The optical field is described as usual by the field operator $\mathbf{E}(\mathbf{r}, t)$. The dynamics of the combined system is governed by the Hamiltonian

$$
\mathcal{H}_{0}=\mathcal{H}_{s}+\mathcal{H}_{m}+\mathcal{V}
$$

where $\mathcal{H}_{s}$ and $\mathcal{H}_{m}$ describe the evolution of the Schrödinger atomic matter-wave field and of the Maxwell light field, respectively, while $\mathcal{V}$ is the interaction between these two fields, typically the electric dipole interaction. It is not necessary to give more details about the specific system at hand at this point.

We now turn to the description of the detector. It consists of two parts: The first one is a conventional optical photodetector that emits photoelectrons when absorbing photons from the light field. In addition, there is also a matter-wave detector. It consists of a tightly focussed laser beam that can ionize atoms, thereby annihilating them and producing a ion-electron pair. The detection of the mutual coherence properties of the coupled MaxwellSchrödinger fields is obtained from the correlations between the electron emitted by these two detectors.

Following the original work of Glauber [12], we describe the photodetector as consisting of two-level atoms whose excited state is in the continuum, corresponding to photoionization. We call it the Maxwell detector. Its interaction with the system is decribed as usual by the electric dipole interaction Hamiltonian

$$
\mathcal{V}_{m}=-\mathbf{d} \cdot \mathbf{E}\left(\mathbf{r}_{m}\right)
$$

where $\mathbf{d}$ is the atomic electric dipole moment between the detector ground electronic state and the continuum 
state, and $\mathbf{r}_{m}$ is the location of the detector, assumed to be punctual for simplicity.

The interaction between the system and the Schrödinger detector, also assumed to be punctual at location $\mathbf{r}_{s}$, is

$$
\mathcal{V}_{s}=\hbar \sum_{i} \Omega_{i}\left(\mathbf{r}_{s}\right)\left[\Psi_{i}^{\dagger}\left(\mathbf{r}_{s}\right) \Psi\left(\mathbf{r}_{s}\right) e^{-i \omega_{L} t}+h . c .\right]
$$

Here $\Psi$ and $\Psi_{i}$ are the field operators associated with atoms in the sample and the continuum state $|i\rangle$, respectively, $\Omega_{i}$ is a coupling constant proportional to the electromagnetic field producing the ionization and $\omega_{L}$ is the laser field frequency. The assumption of a punctual photodetector is well justified for ultracold atomic samples, in which case the ionizing laser can easily be focussed to a spot much smaller than a characteristic atomic de Broglie wavelength.

The Hamiltonian describing the total interaction between the detector and the system is

$$
\mathcal{V}_{d}=\mathcal{V}_{m}+\mathcal{V}_{s}
$$

and the mutual coherence of the optical and atomic fields is determined to lowest order by measuring the joint counts of the Maxwell and Schrödinger detectors, i.e., the joint probability to count one electron

\section{CORRELATIONS BETWEEN DETECTED SIGNALS}

To lowest order, the probability amplitude for the joint detection of electons from the Schrödinger and Maxwell detectors is given by second-order perturbation theory in $\mathcal{V}_{d}$. To that order, the transition probability from the system initial state $|i\rangle$ to a final state $|f\rangle$ in a time interval $\Delta t$ is

$$
-\frac{1}{\hbar^{2}} \int_{t}^{t+\Delta t} d t^{\prime \prime} \int_{t}^{t+\Delta t} d t^{\prime}\left\langle f\left|\mathcal{V}_{d}\left(t^{\prime \prime}\right) \mathcal{V}_{d}\left(t^{\prime}\right)\right| i\right\rangle
$$

where $t^{\prime \prime}>t^{\prime}$, and the detector interaction $\mathcal{V}_{d}$ is now expressed in the interaction picture

$$
\mathcal{V}_{d}(t) \rightarrow e^{i\left(\mathcal{H}_{0}+\mathcal{H}_{d}\right) t} \mathcal{V}_{d}(t) e^{-i\left(\mathcal{H}_{0}+\mathcal{H}_{d}\right) t} .
$$

$\mathcal{H}_{d}$ being the detector Hamiltonian.

When it comes to measuring the cross-correlations between the optical and atomic fields, the relevant terms are of course those involving one electron each emitted by the Schrödinger and Maxwell detectors. In Eq. (6), these are the terms involving cross-products of the detector-system coupling Hamiltonians $\mathcal{V}_{s}$ and $\mathcal{V}_{m}$, that is,

$$
\begin{aligned}
- & \frac{1}{\hbar^{2}} \int_{0}^{t} d t^{\prime \prime} \int_{0}^{t} d t^{\prime} \\
& {\left[\left\langle f\left|\mathcal{V}_{m}\left(t^{\prime \prime}\right) \mathcal{V}_{s}\left(t^{\prime}\right)\right| i\right\rangle+\left\langle f\left|\mathcal{V}_{s}\left(t^{\prime \prime}\right) \mathcal{V}_{m}\left(t^{\prime}\right)\right| i\right\rangle\right] }
\end{aligned}
$$

with $t^{\prime \prime}>t^{\prime}$.

The probability to jointly excite the Maxwell and Schrödinger detectors is therefore

$$
\begin{aligned}
& P(\Delta t) \simeq \frac{1}{\hbar^{4}} \sum_{\{f\}} \mathcal{R}_{m}\left(f_{m}\right) \mathcal{R}_{s}\left(f_{s}\right) \\
& \times \mid \int_{t}^{t+\Delta t} d t^{\prime \prime} \int_{t}^{t+\Delta t} d t^{\prime}\left[\left\langle f\left|\mathcal{V}_{m}\left(t^{\prime \prime}\right) \mathcal{V}_{s}\left(t^{\prime}\right)\right| i\right\rangle\right. \\
& \left.+\left\langle f\left|\mathcal{V}_{s}\left(t^{\prime \prime}\right) \mathcal{V}_{m}\left(t^{\prime}\right)\right| i\right\rangle\right]\left.\right|^{2}, \quad t^{\prime \prime}>t^{\prime}
\end{aligned}
$$

The sum is on a complete set of final states $\{f\}=\left\{\left|f_{0}\right\rangle \otimes\right.$ $\left.\left|f_{m}\right\rangle \otimes\left|f_{s}\right\rangle\right\}$, where $\left\{\left|f_{0}\right\rangle\right\}$ is a complete set of states of the system, and $\mathcal{R}_{m}\left(f_{m}\right)$ and $\mathcal{R}_{s}\left(f_{s}\right)$ are the sensitivities of the Maxwell and Schrödinger detectors, respectively, associated with the final states $\left|f_{m}\right\rangle$ and $\left|f_{s}\right\rangle$ of these detectors. This sum results from the fact that we are not interested in the final state of the system. It allows one to introduce a closure relation and to reexpress Eq. (9) as

$$
\begin{aligned}
& P(\Delta t) \simeq \frac{1}{\hbar^{2}} \int_{t}^{t+\Delta t} d t_{1} \int_{t}^{t+\Delta t} d t_{2} \int_{t}^{t+\Delta t} d t_{3} \int_{t}^{t+\Delta t} d t_{4} \\
& \times\left[\eta_{s}\left(\mathbf{r}_{s}, t_{4}, t_{3}\right) \eta_{m}\left(\mathbf{r}_{m}, t_{2}, t_{1}\right)\right. \\
& \times\left\langle E^{-}\left(\mathbf{r}_{\mathbf{m}}, t_{2}\right) \Psi^{\dagger}\left(\mathbf{r}_{s}, t_{4}\right) \Psi\left(\mathbf{r}_{s}, t_{3}\right) E^{+}\left(\mathbf{r}_{m}, t_{1}\right)\right\rangle \\
& +\eta_{s}\left(\mathbf{r}_{s}, t_{1}, t_{2}\right) \eta_{m}\left(\mathbf{r}_{m}, t_{4}, t_{3}\right) \\
& \left.\times\left\langle\Psi^{\dagger}\left(\mathbf{r}_{s}, t_{2}\right) E^{-}\left(\mathbf{r}_{m}, t_{4}\right) E^{+}\left(\mathbf{r}_{\mathbf{m}}, t_{3}\right) \Psi\left(\mathbf{r}_{s}, t_{1}\right)\right\rangle\right]
\end{aligned}
$$

where

$$
\begin{aligned}
& \eta_{s}\left(\mathbf{r}_{s}, t, t^{\prime}\right)=\frac{1}{\hbar} e^{i \omega_{L}\left(t-t^{\prime}\right)} \sum_{i} \sum_{f_{s}} \mathcal{R}_{s}\left(f_{s}\right)\left|\Omega_{i}\left(\mathbf{r}_{s}\right)\right|^{2} \\
& \times\left\langle i_{s}\left|\Psi_{i}\left(\mathbf{r}_{s}, t\right)\right| f_{s}\right\rangle\left\langle f_{s}\left|\Psi_{i}^{\dagger}\left(\mathbf{r}_{s}, t^{\prime}\right)\right| i_{s}\right\rangle
\end{aligned}
$$

and

$$
\eta_{m}\left(\mathbf{r}_{m}, t, t^{\prime}\right)=\frac{1}{\hbar} \sum_{f_{m}} \mathcal{R}_{m}\left(f_{m}\right)\left\langle i_{m}|d(t)| f_{m}\right\rangle\left\langle f_{m}\left|d\left(t^{\prime}\right)\right| i_{m}\right\rangle
$$

are the detector sensitivities, and $d(t)$ is the atomic dipole operator in the interaction picture.

We have neglected contributions involving the product of two creation and annihilation operators. This follows from the implicit assumptions that the Maxwell detector is initially in its ground state, and that in the Schrödinger field detector any atom in the continuum will be removed from the sample instantaneously. Note also that the statistical properties of the atoms in the detectors do not come into play [13], a consequence of the fact that the lowest order cross-correlations that we consider here involves only one measurement for each of the fields involved.

Equation (10) can be significantly simplified in the case of broadband detection, that is, for situations where the bandwidths of the detectors are much broader than those 
of the detected fields. In this case, and for stationary processes, the Markov approximation can be invoked in the treatment of the detectors. Their responsivities can then be approximated as $\delta$-functions of the time differences $t_{2}-t_{1}$ and $t_{4}-t_{3}$.

Before proceeding with a final expression for the joint counting rates, we observe that expression (10) involves the sum of two normally-ordered correlation functions. This is a consequence of time ordering and of the noncommutivity of the Schrödinger and Maxwell field operators at different times. One possible way to measure each of these correlation functions separately is by using a time-gated detection scheme 14 with nonoverlapping in time windows for the two detectors. Specifically, if the ionizing laser beam of the Schrödinger detector is turned off after a time $t_{s}$ and the photodetector is masked after $t_{m}$, then the coupling of the detectors to the system becomes

$$
\mathcal{V}=\mathcal{V}_{m} \theta\left(t_{m}-t\right)+\mathcal{V}_{s} \theta\left(t_{s}-t\right)
$$

where we can chose for example $t_{s}<t_{m}<\Delta t$. In this particular gating scenario, only the first term in Eq. (10) remains.

Assuming that the spectrum of the Schrödinger and Maxwell fields to be analyzed are centered at $\bar{\omega}_{s}$ and $\bar{\omega}_{m}$, respectively, the probability of registering one count at each detector during the time intervals $t_{s}$ and $t_{m}$ is then

$$
\begin{aligned}
& \mathcal{P}\left(t_{s}, t_{m}\right)=\eta_{m}\left(\mathbf{r}_{m}, \bar{\omega}_{m}\right) \eta_{s}\left(\mathbf{r}_{s}, \bar{\omega}_{s}\right) \int_{0}^{t_{m}} d t_{2} \int_{0}^{t_{s}} d t_{1} \\
& \times\left\langle E^{(-)}\left(\mathbf{r}_{m}, t_{2}\right) \Psi^{\dagger}\left(\mathbf{r}_{s}, t_{1}\right) \Psi\left(\mathbf{r}_{s}, t_{1}\right) E^{(+)}\left(\mathbf{r}_{m}, t_{2}\right)\right\rangle
\end{aligned}
$$

where $\eta_{m}\left(\mathbf{r}_{m}, \bar{\omega}_{m}\right)$ and $\eta_{s}\left(\mathbf{r}_{s}, \bar{\omega}_{s}\right)$ are the Fourier transforms of the detector Maxwell and Schrödinger responsivities. Hence the counting rate is simply propotional to the joint correlation function of the fields,

$$
\begin{aligned}
& w\left(\mathbf{r}_{s} t_{s}, \mathbf{r}_{m} t_{m}\right)=\frac{\partial^{2}}{\partial t_{s} \partial t_{m}} \mathcal{P}\left(t_{s}, t_{m}\right)=\eta_{m}\left(\mathbf{r}_{m}, \bar{\omega}_{m}\right) \eta_{s}\left(\mathbf{r}_{s}, \bar{\omega}_{s}\right) \\
& \times\left\langle E^{(-)}\left(\mathbf{r}_{m}, t_{m}\right) \Psi^{\dagger}\left(\mathbf{r}_{s}, t_{s}\right) \Psi\left(\mathbf{r}_{s}, t_{s}\right) E^{(+)}\left(\mathbf{r}_{m}, t_{m}\right)\right\rangle .
\end{aligned}
$$

For $t_{m}<t_{s}<\Delta t$, it is only the second term in Eq. (10) that persists. It is straightforward to modify the final form (15) of the counting rate accordingly.

Equation (15) is similar to the double-photocount result in conventional optics. This is of course not an accident, since the only difference is that instead of having two photodetectors, we have replaced one of them by a matter-wave detector. However, there is an essential difference since this new detection scheme allows one to measure joint coherence properties of the optical and matter fields. In addition, it is obviuous that the proposed detection scheme can readily be generalized to measure higher-order correlatinos of these fields. In that case, though, the fermionic quantum statistics of the ions and electrons resulting from the photoionization of the bosonic matter-wave field must be handled properly [13. This will be the subject of a future publication.
To see how the lowest-order cross-coherence measurement of Eq. (15) works in practice, we illustrate it for the case of a simple example.

\section{EXAMPLE}

The simplest situation one can consider is that of a linear coupling between the optical and matter-wave field. Of course, we should keep in mind such a coupling is not possible in principle, due to the conservation of the number of particles. Instead, all couplings must be at least bilinear in the Schrödinger field operators, so that an atom annihilated in one state is then created in another state. However, the advent of Bose-Einstein condensates with a large number of atoms in a single, macroscopically populated quantum state, allows one to describe that state as a c-number. This is analogous to the undepleted, classical field approximation in quantum optics. In that case, the bilinear matter-wave part of the interaction Hamiltonian, which involves both the condensate mode and some other side mode, approximately reduces to a linear form only (see e.g. [8, 15]). With this kind of situation in mind, we then consider a matter-light interaction Hamiltonian of the general form

$$
\begin{aligned}
\mathcal{H}_{s} & =\sum_{\alpha=1}^{N} \hbar \omega_{\alpha} a_{\alpha}^{\dagger} a_{\alpha}+\sum_{i=1}^{M} \hbar \omega_{i} c_{i}^{\dagger} c_{i} \\
& +\hbar \sum_{i \alpha}\left[g_{i}^{(1)} a_{\alpha}^{\dagger} c_{i}+g_{i}^{(2)} a_{\alpha} c_{i}+\text { H.c. }\right]
\end{aligned}
$$

where $N$ and $M$ are the number of modes in the optical and matter field, respectively, with eigenfrequencies $\omega_{\alpha}$ and $\omega_{i}$. (We use greek indices for the optical field modes and roman letters for the matter waves in this example.) The mode annihilation operators

$$
a_{\alpha}=\int d r \mathbf{u}_{\alpha}^{\star}(\mathbf{r}) \mathbf{E}^{+}(\mathbf{r})
$$

and

$$
c_{i}=\int d r \phi_{i}^{\star}(\mathbf{r}) \Psi(\mathbf{r})
$$

are bosonic optical field mode operators, $\mathbf{u}_{\alpha}(\mathbf{r})$ and $\boldsymbol{\phi}_{i}(\mathbf{r})$ being the corresponding mode functions, $\mathbf{E}^{+}(\mathbf{r})$ is the positive frequency part of the electric field operator, $g_{i}^{(1)}, g_{i}^{(2)}$ are coupling constants, which allow for a parametric amplification type of matter-light coupling as well. These constants are proportional to the order parameter of the condensate ground state, as discussed above.

In addition, we allow for both the optical and the matter modes to be coupled to thermal reservoirs which result in losses at rates $\kappa_{\alpha}$ and $\kappa_{i}$. The Langevin equations describing the evolution of this model system are 16

$$
\frac{d}{d t} \mathbf{x}(t)=-\mathbf{M} \mathbf{x}(t)+\mathbf{B} \boldsymbol{\xi}(t),
$$


where $\mathbf{x}(t)=\left\{a_{\alpha}(t), c_{i}(t)\right\}^{T}, \alpha=\{1, \ldots, N\} ; i=$ $\{1, \ldots, M\}$, and $\mathbf{M}$ is a $(N+M) \times(N+M)$ matrix whose elements depend on the system parameters. The diffusion matrix in Eq. (19) is $\mathbf{D} \equiv \mathbf{B B}^{\dagger}=\operatorname{diag}\left(\bar{n}_{\alpha} \kappa_{\alpha}, \bar{n}_{i} \kappa_{i}\right)$, where $\bar{n}_{\alpha}$ and $\bar{n}_{i}$ are the thermal populations of the reservoir modes. We assume for simplicity that the reservoir operators can be treated in the Markov approximation as $\delta$-correlated white noise with zero expectation value, i.e. $\left\langle\xi_{i}(t)\right\rangle=0$ and $\left\langle\xi_{i}(t) \xi_{j}\left(t^{\prime}\right)\right\rangle=\delta_{i j} \delta\left(t-t^{\prime}\right)$.

For this example, the counting rate of Eq. (15) reduces to a sum over all possible fourth-order cross-correlation functions

$$
\begin{aligned}
& w\left(\mathbf{r}_{s} t_{s}, \mathbf{r}_{m} t_{m}\right)=\sum_{\alpha \beta i j} u_{\alpha}^{\star}\left(\mathbf{r}_{m}\right) u_{\beta}\left(\mathbf{r}_{m}\right) \phi_{i}^{\star}\left(\mathbf{r}_{s}\right) \phi_{j}\left(\mathbf{r}_{s}\right) \\
& \times\left[\left(U^{-1} G\left(t_{s}, t_{m}\right)\left(U^{-1}\right)^{\dagger}\right)_{j \alpha}\left(U^{-1} G\left(t_{m}, t_{s}\right)\left(U^{-1}\right)^{\dagger}\right)_{\beta i}\right. \\
& \left.+\left(U^{-1} G\left(t_{s}, t_{s}\right)\left(U^{-1}\right)^{\dagger}\right)_{j i}\left(U^{-1} G\left(t_{m}, t_{m}\right)\left(U^{-1}\right)^{\dagger}\right)_{\alpha \beta}\right],
\end{aligned}
$$

where $U$ is the matrix of eigenvectors of the system matrix $\mathbf{M}$, so that

$$
U^{-1} \mathbf{M} U=\operatorname{diag}\left(\lambda_{k}\right), k=\{1, \ldots, N+M\},
$$

where $\left\{\lambda_{i}\right\}$ are the eigenvalues of $\mathbf{M}$, and

$$
\begin{aligned}
G_{i j}(t, s) & =\left(U \mathbf{D} U^{\dagger}\right)_{i j} \frac{e^{-\lambda_{i}(t-s)}}{\lambda_{i}+\lambda_{j}^{\star}} & t \geq s, \\
G_{i j}(t, s) & =\left(U \mathbf{D} U^{\dagger}\right)_{i j} \frac{e^{-\lambda_{j}^{\star}(s-t)}}{\lambda_{i}+\lambda_{j}^{\star}} & t<s .
\end{aligned}
$$

The two terms in square brackets in Eq. (20) correspond to the stationary value of the counting rate and to the exponential approach to that value. These expressions follow directly from the stationary solution of Eq. (19),

$$
\mathbf{x}(t)=\int_{-\infty}^{t} e^{-\mathbf{M}\left(t-t^{\prime}\right)} \mathbf{B} \boldsymbol{\xi}\left(t^{\prime}\right) d t^{\prime}
$$

where $\mathbf{x}(0)=\left\{a_{\alpha}, c_{i}\right\}^{T}$.

The possibility to extract particular mode correlation functions from these general expressions depends on the specifics of the problem. To gain some insight, we consider the two-mode problem with the drift matrix

$$
\mathbf{M}=\left(\begin{array}{cc}
\kappa_{1} / 2 & i g \\
i g & \kappa_{2} / 2
\end{array}\right)
$$

and diffusion matrix

$$
\mathbf{D}=\left(\begin{array}{cc}
\bar{n}_{1} \kappa_{1} & 0 \\
0 & \bar{n}_{2} \kappa_{2}
\end{array}\right)
$$

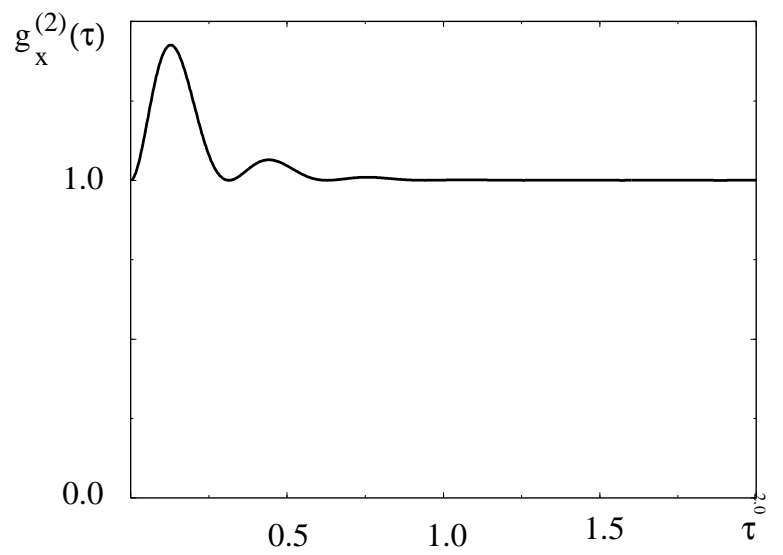

FIG. 1. Time dependence of the normalized stationary cross-correlation function $g_{x}^{(2)}(\tau)$ of the optical and matter-wave modes for $g=10 ., \kappa_{1}=6 ., \kappa_{2}=6 ., \bar{n}_{1}=0.1$ and $\bar{n}_{2}=0.1$

The normalized stationary cross-correlation function $g_{x}^{(2)}(\tau)$ of the optical and matter-wave modes is shown in Fig.1 as a function of the time difference $\tau=t_{m}-t_{s}$. As expected, for this case of linear system with positive diffusion coefficients, $g_{x}^{(2)}(\tau)$ exhibits bunching and $\lim _{\tau \rightarrow \infty} g_{x}^{(2)}(\tau)=1$. The oscillations correspond to the fact that for the chosen parameters the eigenfrequencies of the system are complex, with a real part smaller than the imaginary part.

\section{DISCUSSION AND CONCLUSIONS}

In this paper, we have generalized the familiar approach to optical detection and coherence theory to deal with situations involving coupled Maxwell and Schrödinger fields. We have proposed a concrete detection scheme that allows one to experimentally determine cross-correlation functions and the mutual coherence of these fields. We have concentrated only on the lowestorder cross-correlation, but the scheme is obviously easily generalized to measure higher-order correlations. In that latter case, though, proper care must be taken of the quantum statistics of the detected signal. This topic, where the fact that atoms are actually composite bosons (or fermions) plays a central role, will be discussed in a future publication.

We have illustrated our detection scheme on a simple case of linear coupling between an atomic matter wave and an optical field. The results in that case are quite similar to the situation involving only optical fields. This is a direct consequence of the linearization procedure leading to our model Hamiltonian. More realistic situations will require the use of an interaction which is at least bilinear in the matter-wave field, in which case major differences can be expected, for instance in situations involving small condensates.

The detection scheme presented here, as well as its generalizations, are expected to find applications in a 
number of situations involving e.g. the optical control and manipulation of matter waves, nonlinear atom optics, the generation and detection of quantum entanglement between light and matter, and other applications of quantum atom optics. Note however that some of our simplifying assumptions, such as e.g. the model a punctual detector, only hold provided that the ionizing laser can be focused to a spot small compared to an atomic de Broglie wavelength. As such, it is geared toward ultracold samples. It would not be difficult to expand our scheme to detectors of finite size, but in that case, the detected photocounts will involve a convolution with the spatial detector resolution, with a concomitant washing out of the finer spatial structure of the cross-correlations.

\section{ACKNOWLEDGMENTS}

We are indebted to M. G. Moore for numerous discussions and valuable suggestions. This work is supported in part by the U.S. Office of Naval Research under Contract No. 14-91-J1205, by the National Science Foundation under Grant No. PHY98-01099, by the U.S. Army Research Office, and by the Joint Services Optics Program. G. A. P. thanks Fapesp for financial support.

[1] D. M. Stamper-Kurn et al., Phys. Rev. Lett. 80, 2027 (1998).

[2] L. Deng et al., Nature 398, 218 (1999).

[3] M. Kozuma et al., Phys. Rev. Lett. 82, 871 (1999).

[4] J. Stenger et al., Phys. Rev. Lett. 82, 4569 (1999).

[5] S. Inouye et al., unpublished (1999).

[6] M. G. Moore and P. Meystre, Phys. Rev. A 58, 3248 (1998).

[7] L. Kuang, Commun. Theor. Phys. 30, 161 (1998).

[8] M. G. Moore and P. Meystre, Phys. Rev. A 59, R1754 (1999).

[9] M. G. Moore and P. Meystre, condmat/9905425 .

[10] R. Bonifacio, L. D. Salvo, L. M. Narducci, and E. J. D'Angelo, Phys. Rev. A 50, 1716 (1994).

[11] R. Bonifacio and L. D. Salvo, Nucl. Instr. Methods A 50, 360 (1994).

[12] R. Glauber, in Quantum Optics and Electronics, edited by C. de Witt, A. Blandin, and C. Cohen-Tannoudji (Gordan and Breach, New York, 1965).

[13] E. V. Goldstein and P. Meystre, Phys. Rev. Lett. 80, 5036 (1998).

[14] C. Cohen-Tannoudji, J. Dupont-Roc, and G. Grynberg, Atom-Photon Interaction. Basic Processes and Applications (New York : Wiley, New York, 1992).

[15] M. G. Moore, O. Zobay, and P. Meystre, Phys. Rev. A 60, (1999).

[16] P. Meystre and M. S. III, Elements of Quantum Optics (Springer-Verlag, Heidelberg, 1998). 